\title{
Electronic cigarette use and smoking cessation in cohort studies and randomized trials: A systematic review and meta- analysis
}

\author{
Linnea Hedman ${ }^{1,2}$, Maria R. Galanti3,4, Lotta Ryk ${ }^{5}$, Hans Gilljam³, Louise Adermark ${ }^{6,7}$
}

\begin{abstract}
INTRODUCTION The aim of this systematic review and meta-analysis was to assess the association between e-cigarette use and subsequent smoking cessation in cohort studies and randomized controlled trials (RCT).

METHOdS A systematic literature search was finalized 11 November 2019 using EMBASE, Cochrane Library, Scopus, PubMed Health, NICE evidence search, PROSPERO, CRD, PsycInfo, and PubMed including Medline. Inclusion criteria were: reporting empirical results; longitudinal observational design with a minimum of 3 months of follow-up; including general population samples; and allowing for comparison between users and non-users of e-cigarettes. Studies rated as having high risk of bias were excluded. The procedures described by PRISMA were followed, and the quality of evidence was rated using GRADE. RESULTS Twenty-eight longitudinal, peer-reviewed publications from 26 cohort studies, and eight publications from seven RCTs assessing the association between e-cigarette use and smoking cessation were included in this review. A randomeffects meta-analysis based on 39147 participants in cohort studies showed a pooled unadjusted odds ratio (OR) for smoking cessation among baseline e-cigarette users compared with baseline non-users of 0.97 (95\% CI: 0.67-1.40), while the adjusted OR was 0.90 (95\% CI: $0.63-1.27)$. The pooled odds ratio for smoking cessation in RCTs was 1.78 (95\% CI: 1.41-2.25). The evidence for cohort studies was graded as very low and for RCTs as low.

CONCLUSIONS We did not find quality evidence for an association between e-cigarette use and smoking cessation. Although RCTs tended to support a more positive association between e-cigarette use and smoking cessation than the cohort studies, the grading of evidence was consistently low.
\end{abstract}

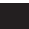

\section{AFFILIATION}

1 Department of Public Health and Clinical Medicine, Division Sustainable Health, The OLIN Unit, Umeå University, Umeå, Sweden 2 Department of Health Sciences, Division of Nursing, Luleå University of Technology, Luleå, Sweden

3 Department of Global Public Health, Karolinska Institutet,

Stockholm, Sweden

4 Centre of Epidemiology and Community Medicine, Stockholm, Sweden

5 Swedish Agency for Health Technology Assessment and Assessment of Social Services (SBU), Stockholm, Sweden 6 Department of Psychiatry and Neurochemistry, Sahlgrenska

Academy, University of Gothenburg, Gothenburg, Sweden 7 Department of Pharmacology, Sahlgrenska Academy, University of Gothenburg, Gothenburg, Sweden
\end{abstract}

\section{CORRESPONDENCE TO}

Linnea Hedman. Department of Public Health and Clinical Medicine, Division of Sustainable Health, The OLIN unit, Umeå University, 901 87, Umeå, Sweden. E-mail: linnea.hedman@ norrbotten.se ORCID ID: https:// orcid.org/0000-0002-1630-3167

\section{KEYWORDS}

electronic cigarettes, smoking cessation, quit smoking, systematic review, cohort, randomized controlled trial

Received: 20 May 2021

Revised: 1 September 2021

Accepted: 15 September 2021

\section{INTRODUCTION}

During the last two decades, electronic cigarettes (e-cigarettes) have been the subject of a heated debate and polarized responses within the field of 
tobacco control and prevention ${ }^{1-3}$. On the one hand, it has been suggested that e-cigarettes may present a less harmful alternative to combustible cigarettes that potentially could lead to beneficial effects on individual and public health ${ }^{4,5}$. On the other hand, e-cigarette use is associated with increased risk for smoking initiation among non-smokers ${ }^{6}$. In addition, there is no conclusive evidence that e-cigarette use facilitates smoking cessation ${ }^{7}$ or contributes to harm reduction ${ }^{8}$. The results of systematic reviews of the association between e-cigarette use and smoking cessation have shown inconsistent results. Two of the earlier systematic reviews published between 2014 and 2016, concluded that there was an association between e-cigarette use and smoking cessation ${ }^{9,10}$, while another review found that e-cigarette use reduced the likelihood of smoking cessation ${ }^{11}$. However, these reviews included few studies and thus based the conclusions on small samples. More recent systematic reviews have concluded that it was not possible to determine whether there was an association between e-cigarette use and subsequent smoking cessation ${ }^{12,13}$. The publication rate of studies about e-cigarette use and smoking cessation is rapidly growing and in the most recently updated systematic review from the Cochrane Library, the evidence level was rated as moderate ${ }^{7}$ compared to low in the previous editions ${ }^{9,14}$. However, some of these reviews include publications with study designs that are subject to numerous limitations, such as studies with a cross-sectional design or present pooled data from cohort studies and randomized controlled trials (RCT) in the same analysis. The distinction between observational and experimental studies is crucial in this domain, because of the potential of bias by confounding in the former and because of the likely selection of motivated smokers in the latter. The aim of this systematic review and meta-analysis was to assess the association between e-cigarette use and subsequent smoking cessation in cohort studies and RCTs, respectively.

This review is based on data previously published as a report from The Swedish Agency for Health Technology Assessment and Assessment of Social Services (SBU) (Report 312, SBU 2020/431) ${ }^{15}$. A summary of the protocol was published in Swedish at the SBU website in February 2019 (https://www. sbu.se/sv/publikationer/forstudie-sammanfattning/ forstudie-tobaksprevention/) and the complete protocol can be requested (from registrator@sbu.se).

\section{METHODS}

\section{Research question}

This review had the following research question: 'Is there evidence for an association between e-cigarette use and subsequent smoking cessation among people smoking tobacco in the beginning of the study period and do the results differ between cohort studies and RCTs?'. The PICO (Population, Intervention, Comparison, and Outcome) frame used to establish the eligibility criteria is presented in Tables 1 and 2.

\section{Table 1. PICO frame in cohort studies}

$\begin{array}{ll}\text { Population } & \text { General population samples of current smokers, any age. } \\ \text { Intervention/exposure } & \text { Self-reported current or ever use of e-cigarettes at baseline, with or without nicotine. } \\ \text { Comparison group } & \text { Self-reported non-use of e-cigarettes. } \\ \text { Outcome } & \text { Self-reported abstinence from combustible tobacco products at follow-up. }\end{array}$

Table 2. PICO frame in randomized controlled trials 


\section{Literature search}

A comprehensive literature search of EMBASE, Cochrane library, Scopus, Pubmed Health, NICE evidence search, PROSPERO, CRD (DARE), PsycInfo and PubMed including Medline was conducted in December 2018 and updated 11 November 2019. Search terms were based on the following keywords: [electronic cigarettes, vaping, vaporized nicotine, electronic nicotine delivery system] combined with [cigarettes, smoking, combustible tobacco]. We also asked researchers in the field to provide lists of relevant articles and we read reports from Swedish authorities and Health Technology Assessment organizations for background material. Details on the literature search can be found in Supplementary file Table S1.

\section{Inclusion and exclusion criteria}

Inclusion criteria were: published in peer review journals between 1 January 1990 and 11 November
2019; longitudinal design or randomized controlled trial, with at least one baseline and one follow-up measure; included a parallel control group; at least 3 months follow-up; presentation of the results in a way that could allow the calculations of risk ratios or equivalent association measures (e.g. odds ratios); and written in English, Swedish, Norwegian or Danish. Exclusion criteria were: conference abstracts, book chapters or articles not reporting empirical results; and studies based on selected samples and not representative of the underlying general populations (e.g. military personnel, patients with specific diagnoses, including psychiatric disorders). Studies reporting conflicts of interest were not excluded, but information on conflicts of interest presented in the included articles is presented in Supplementary file Table S2. The study selection processes, and the articles excluded in each step are further outlined in Figure 1. Details on excluded articles can be found in Supplementary file Tables S3 and S4.

Figure 1. Study selection flowchart

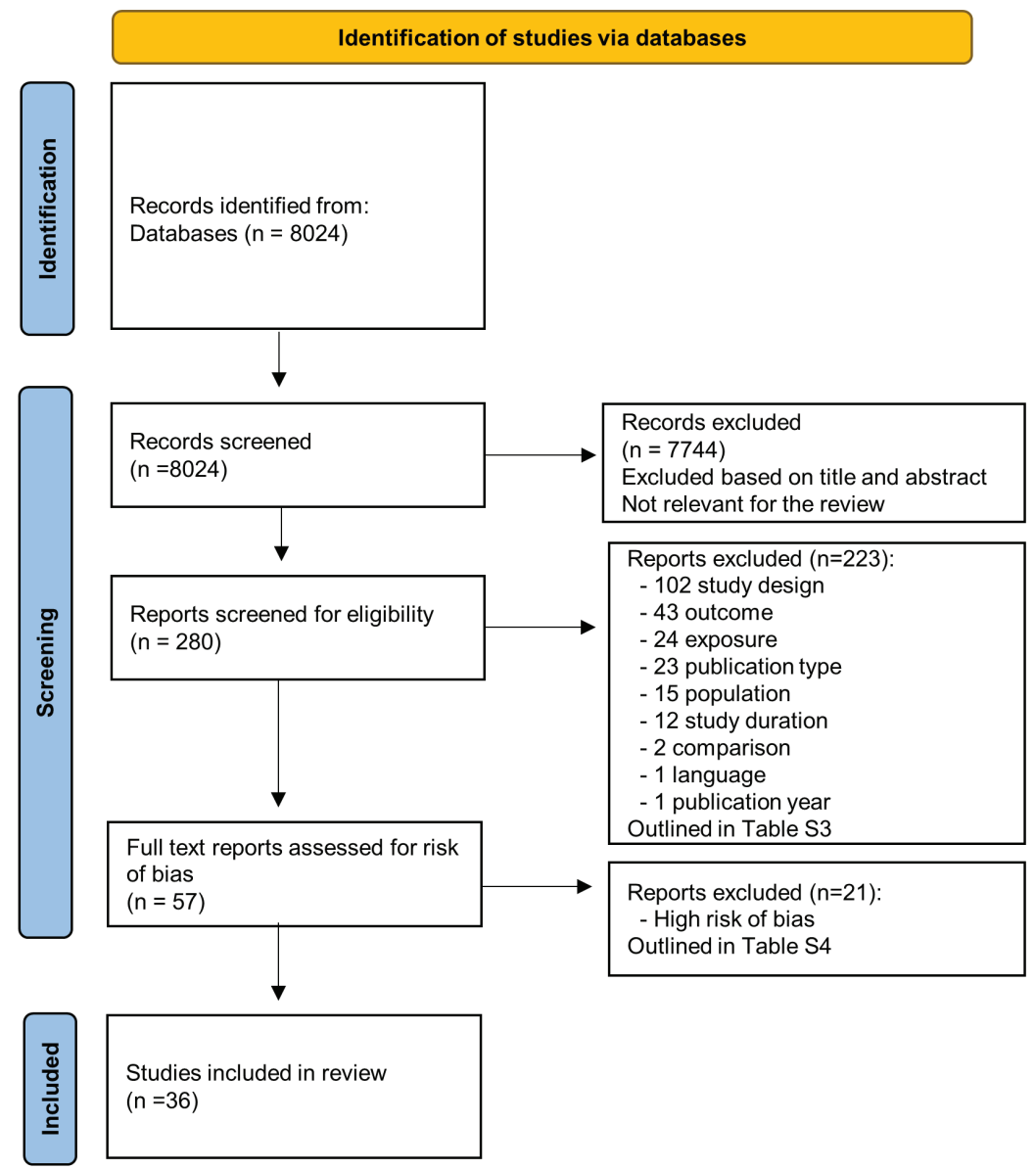

Only longitudinal studies with at least three months study duration and judged to be of low or moderate risk of bias were included in the review. 


\section{Assessment of risk of bias}

The literature was assessed using a priori established protocols, and reported according to the Preferred Reporting Items for Systematic Reviews and MetaAnalyses (PRISMA; http://www.prisma-statement. org/). The screening of the publications was performed using established protocols based on Cochrane's templates: ROBINS-I and ROBINS-E for the cohort studies and the SBU risk-of-bias tool for randomized trials based on the Cochrane RoB 2 template for the RCTs (https://www.riskofbias. info/). These templates have been developed by building upon tools for risk-of-bias assessment of randomized trials, diagnostic test accuracy studies and observational studies of interventions, and has been developed by members of the Cochrane Bias Methods Group and the Cochrane Non-Randomized Studies of Interventions Methods Group ${ }^{16}$. The title and abstract of each publication were initially reviewed by two of the authors. Disagreements were resolved by a third reviewer. None of the reviewers had any conflicts of interest regarding the rated publications.

Publications read in full text but rated as not eligible for this review are listed in Supplementary file Table S3. Those considered eligible underwent a risk-of-bias assessment independently by two of the authors. Only prospective studies with a low or moderate risk-of-bias in all domains were included in the analysis (Figure 1). For observational studies, the categories were: selection, exposure, judgement, drop-out rate, or reporting. For RCTs, the categories were: randomization, deviation from planned intervention, attrition, measurement of outcome, or reporting. Articles excluded due to high risk-of-bias are presented in Supplementary file Table S4.

\section{Data extraction and statistical analysis}

Main features extracted from the selected studies were: authors, date of publication, study design, subjects and sample size, and main quantitative results (Supplementary file Tables S5 and S6). We included studies where it was possible to retrieve either absolute numbers or percentages for each group (unadjusted analysis), or where the study presented the results as both adjusted and unadjusted odds ratios. All analyses were conducted according to the a priori analysis plan, with two main outcomes: nonuse of combustible tobacco and non-use for at least
30 days at the time of the follow-up. Unadjusted and adjusted data were analyzed separately, and studies where odds ratios could not be calculated were not included in the meta-analyses but contributed to the narrative summary.

To assess the association between e-cigaretteuse and subsequent smoking cessation, relevant data were combined in a random-effects metaanalysis, combined with inverse variance using Review Manager 5.3 [Review Manager (RevMan) Version 5.3. Copenhagen: The Nordic Cochrane Centre, The Cochrane Collaboration, 2014]. We also considered the following subgroups: duration of follow-up ( $\leq 6$ months or $>6$ months), age $(<18$ years or $\geq 18$ years, based on the legal age for purchasing tobacco products in many countries) and sex (male or female). We also conducted separate analyses for cohort and RCT studies. Subgroups for further analysis were defined a priori. During the evaluation, several studies were shown to build on the same or overlapping cohorts. In these cases, only the most complete data set from the overlapping study populations was used in our meta-analysis. If a study presented several sets of adjusted data, with different numbers of confounders, the least adjusted model that contained the variables sex, age and socioeconomic factors was chosen. This was done to make the estimates included in the analysis as comparable as possible. Some observational studies measured the exposure (e-cigarette use) at baseline, but some studies measured the exposure at followup and the meta-analyses are presented separately for these two different methods. A putative publication bias was assessed using a funnel plot.

\section{Grading of evidence}

We followed the GRADE guidelines to appraise the strength of evidence for the presence of the association, resulting from the meta-analysis ${ }^{17-20}$. In this approach, direct evidence from longitudinal studies starts at high certainty $(\oplus \oplus \oplus \oplus)$ and is downgraded based on risk-of-bias, indirectness, imprecision, and inconsistency to levels of moderate $(\oplus \oplus \oplus)$, low $(\oplus \oplus)$ and very low $(\oplus)$ certainty of evidence. Both unadjusted and adjusted estimates were taken into account when grading the evidence. Grading was performed separately for observational studies and RCTs. 


\section{RESULTS}

\section{Description of the publications included in the review}

In total, 28 peer-reviewed publications based on 26 cohort studies, and 8 publications from 7 RCTs were included. Of the RCT studies, data from one of the trials are presented in two of the publications, and one publication is included narratively.

\section{Cohort studies}

The included studies are described in detail in the Supplementary file Table S5. Twenty of the cohort studies were performed in the $\mathrm{USA}^{21-42}$, one in Canada ${ }^{43}$, and five in Europe ${ }^{44-48}$. The study populations included on average $53 \%$ females, varying from $43 \%{ }^{34}$ to $100 \%^{23}$. Three of the cohorts included individuals $<18$ years ${ }^{21,30,37,43}$, two included a population with a mixed age $\operatorname{span}^{33,48}$, while all other articles included adults aged $\geq 18$ years at baseline.

The educational level of participants $>18$ years was reported in 13 of the studies ${ }^{24-26,28,29,32-35,38-40,42}$. On average, $60 \%$ of the participants in these studies had attended school for 12 years or longer. The educational level varied from $11 \%$ with at least college level ${ }^{40}$ to $88 \%$ with at least four years at college $\mathrm{e}^{24}$. The other studies did not report educational level. Three studies collected data through questionnaires distributed during school hours ${ }^{21,30,37,43}$, five studies used questionnaires distributed by mail or in other ways ${ }^{29,33,35,39,46}$, eleven studies collected data through in-person interviews ${ }^{23,26,28,31,32,34,36,40,45,47}$, and the others used web-based surveys ${ }^{24,25,27,38,42,48}$. The follow-up time varied from 6 months to 4 years.

Most studies defined smoking at baseline based on smoking during the past 30 days, while eight studies had other definitions of tobacco smoking, for instance ever smokers, established smokers, tobacco smokers, or as having smoked at least 100 cigarettes in their lifetime and now smoking at least on some days ${ }^{21,24,27,29,33,34,39,48}$. In two of the studies, the participants had plans to quit smoking ${ }^{35,40}$, in one study $28-50 \%$ of the participants intended to quit smoking $^{29}$, and in one study the participants reported no intention to quit in the next 30 days $^{28}$. The other studies presented no information on the participants' intention or motivation to quit smoking.

The exposure group is described as e-cigarette- users in most studies s $^{23,24,26,28,31,32,34-36,41-48}$, as current e-cigarette users in seven studies ${ }^{24,28,29,42,43,46,48}$, and as ever e-cigarette users in two studies ${ }^{30,39}$. In two studies, the exposure group is referred to as ENDS users (electronic nicotine delivery systems), including all types of electronic vapor products $^{27,33}$. The control group is referred to as never e-cigarette users ${ }^{21,25,27,29,30,38,39}$, non-users of e-cigarettes ${ }^{23,24,26,28,31,32,34-36,41-48}$, never ENDS users ${ }^{33}$, or users of nicotine replacement therapy (NRT), varenicline, buproprion, or no aid used ${ }^{40}$.

\section{Randomized controlled trials}

The included studies are described in detail in the Supplementary file Table S6. Of the RCTs, two were performed in the USA ${ }^{49,50}$, three in Europe ${ }^{51-53}$, two in New Zealand ${ }^{54-56}$, and one in Korea ${ }^{57}$. On average, the trials included $42 \%$ women, with a range between $0 \%{ }^{57}$ to $66 \%{ }^{56}$. All RCTs included participants aged $>18$ years at baseline. One study reported that $25 \%$ of the participants had college education or higher ${ }^{50}$, one reported that $64 \%$ of the participants had attended school for at least 12 years $^{56}$, while the other studies did not report educational level. All studies collected data through in-person interviews. The follow-up time varied between 3 and 12 months.

Five studies defined tobacco smoking at baseline based on smoking during the past 30 days ${ }^{49,52-55,57}$ while the other studies employed other definitions such as current cigarette smokers of more than two cigarettes per day, having smoked at least once in the last seven days ${ }^{50}$, adult smokers ${ }^{51}$, or tobacco smokers ${ }^{56}$. The participants in the RCTs wanted to quit smoking ${ }^{54-57}$, were motivated to quit smoking ${ }^{53}$, were recruited from smoking cessation programs $\mathrm{s}^{51}$, were worried about the health effects of smoking and were willing to try new alternatives ${ }^{49}$, or were waiting for surgery ${ }^{50}$. In only one study, wanting to quit smoking was not an inclusion criterion ${ }^{52}$.

The exposure group was described as assigned to e-cigarette use in all studies, and the participants received e-cigarettes free-of-charge. In three trials, presented in four publications, participants were assigned either e-cigarettes with or without nicotine, but in the present analyses these groups were combined ${ }^{53-56}$. The other studies included e-cigarettes with nicotine only ${ }^{49-52,57}$. The control groups included participants not receiving 
e-cigarettes free-of-charge ${ }^{49,52}$, participants with access to nicotine patches or gum free-ofcharge $\mathrm{e}^{50,54-57}$, or participants offered telephone counseling to quit smoking ${ }^{53}$.

\section{Smoking cessation among e-cigarette users and non-users in cohort studies}

Unadjusted data were presented in 18 of the included studies, including a total of 39147 participants (Figure 2). Among baseline smokers, the unadjusted odds ratio for smoking cessation ranged between 0.30 and 3.00 across studies that measured e-cigarette use at baseline, and 0.21 to 1.38 across studies measuring e-cigarette use retrospectively at followup. When combining the data in a random-effects meta-analysis, the pooled unadjusted odds ratio for smoking cessation was 0.97 (95\% CI: 0.67-1.40) and 1.09 (95\% CI: 0.90-1.33), respectively (Figure 2).

Adjusted results were presented in 14 studies (Figure 3). Adjustments were made for sex (11/14), age (13/14) and socioeconomic factors (13/14), but there was a large heterogeneity with respect to how these adjustments were made. The adjusted odds ratio for smoking cessation ranged between 0.30 and 2.09 for studies that measured e-cigarette use at baseline, and between 0.40 and 2.02 for studies that measured e-cigarette use retrospectively at followup. The pooled adjusted odds ratio for smoking cessation was 0.90 (95\% CI: 0.63-1.27) for studies measuring e-cigarette use at baseline and 1.12 (95\%

Figure 2. E-cigarette use and subsequent smoking cessation in cohort studies, unadjusted analyses

Odds Ratio

Study or Subgroup log[Odds Ratio]

SE Weight IV, Random, $95 \% \mathrm{Cl}$ Exposure measured at baseline

Al-Delaimy 2015

Barrington-Trimis 2019

Brose 2015

Gomajee 2019

Grana 2014

Hammond 2017

Kasza 2018

Kurti 2018

Leventhal 2016

Manzoli 2017

Pasquereau 2017

Piper 2019

Weaver 2018

Subtotal $(95 \% \mathrm{Cl})$ $\begin{array}{lll}-1.204 & 0.3537 \quad 4.7 \%\end{array}$

$\begin{array}{lll}0.571 & 0.3283 \quad 4.9 \%\end{array}$

$\begin{array}{lll}-0.3857 & 0.1994 \quad 6.4 \%\end{array}$

$\begin{array}{lll}0.708 & 0.0757 \quad 7.4 \%\end{array}$

$\begin{array}{lll}-0.3425 & 0.3609 & 4.6 \%\end{array}$

$-0.734 \quad 0.4467 \quad 3.8 \%$

$\begin{array}{lll}-0.3857 & 0.09 & 7.3 \%\end{array}$

$\begin{array}{lll}-0.462 & 0.2192 \quad 6.1 \%\end{array}$

$-0.2107 \quad 0.542 \quad 3.1 \%$

$0.157 \quad 0.1876 \quad 6.5 \%$

$0.4574 \quad 0.2037 \quad 6.3 \%$

$\begin{array}{lll}1.0986 & 0.6434 \quad 2.5 \%\end{array}$

$0.4121 \quad 0.2418 \quad 5.9 \%$

$69.6 \%$
Odds Ratio

IV, Random, $95 \% \mathrm{Cl}$

Heterogeneity: $\mathrm{Tau}^{2}=0.35 ; \mathrm{Chi}^{2}=132.90, \mathrm{df}=12(\mathrm{P}<0.00001) ; \mathrm{I}^{2}=91 \%$

Test for overall effect: $Z=0.16(P=0.87)$

Exposure measured at follow-up

$\begin{array}{lrrrr}\text { Benmarhnia 2018 } & 0.0198 & 0.1176 & 7.1 \% & 1.02[0.81,1.28] \\ \text { Berry 2019b } & 0.3221 & 0.1493 & 6.9 \% & 1.38[1.03,1.85] \\ \text { Curry 2018 } & -1.5606 & 0.7322 & 2.1 \% & 0.21[0.05,0.88] \\ \text { Harlow 2018 } & 0 & 0.0951 & 7.3 \% & 1.00[0.83,1.20] \\ \text { Zhuang 2016 } & 0.1823 & 0.13 & 7.0 \% & 1.20[0.93,1.55] \\ \text { Subtotal }(95 \% \mathrm{Cl}) & & & \mathbf{3 0 . 4 \%} & \mathbf{1 . 0 9}[0.90,1.33]\end{array}$

Subtotal $(95 \% \mathrm{Cl})$

$0.30[0.15,0.60]$

$1.77[0.93,3.37]$

$0.68[0.46,1.01]$

$2.03[1.75,2.35]$

$0.71[0.35,1.44]$

$0.48[0.20,1.15]$

$0.68[0.57,0.81]$

$0.63[0.41,0.97]$

$0.81[0.28,2.34]$

$1.17[0.81,1.69]$

$1.58[1.06,2.36]$

$3.00[0.85,10.59]$

$1.51[0.94,2.43]$

$0.97[0.67,1.40]$

Heterogeneity: $\mathrm{Tau}^{2}=0.03 ; \mathrm{Chi}^{2}=9.24, \mathrm{df}=4(\mathrm{P}=0.06) ; \mathrm{I}^{2}=57 \%$

Test for overall effect: $Z=0.88(P=0.38)$

Total $(95 \% \mathrm{Cl})$

$100.0 \%$

$0.99[0.78,1.26]$

Heterogeneity: $\mathrm{Tau}^{2}=0.20 ; \mathrm{Chi}^{2}=143.12, \mathrm{df}=17(\mathrm{P}<0.00001) ; \mathrm{I}^{2}=88 \%$

Test for overall effect: $Z=0.10(P=0.92)$

Test for subgroup differences: $\mathrm{Chi}^{2}=0.31, \mathrm{df}=1(\mathrm{P}=0.58), \mathrm{I}^{2}=0 \%$

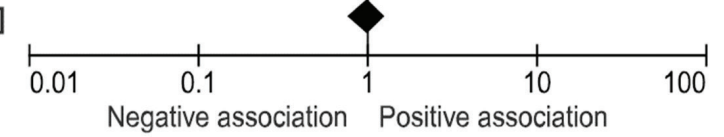

Meta-analysis of unadjusted odds of smoking cessation among e-cigarette users compared with non-e-cigarette users. Data are based on a total of 39147 participants, from 18 non-overlapping studies of low or medium risk of bias. 
CI: 0.54-2.30) for studies measuring e-cigarette use retrospectively at follow-up (Figure 3 ).

Stratified analyses of studies with a short $(\leq 6$ months) or a long ( $>6$ months) follow-up time (Supplementary file Figure S1), studies among adolescents ( $<18$ years) or adults $(\geq 18$ years) (Supplementary file Figure S2), and one study stratified by sex (Supplementary file Figure S3) showed similar results.

\section{Certainty of evidence - cohort studies}

The strength of the evidence concerning the association between e-cigarette use and smoking cessation was deemed to be very low $(\oplus)$ due to risk of bias in any of the categories: selection, exposure, judgement or reporting, inconsistency or imprecision between studies, or that the material was based on few studies or few participants (Table 3). For both the unadjusted and adjusted analyses, the direction of the association was inconsistent.

\section{Smoking cessation among e-cigarette users and non-users in RCTs}

Number or percentage of participants that had quit smoking was presented in all 8 studies, including a total of 3203 participants. One study was included narratively as the outcome was presented as a continuous variable ${ }^{57}$. All included RCTs showed a positive association between e-cigarette use and smoking cessation with odds ratios for smoking cessation ranging between 1.16 and 3.35 (Figure 4). When combining the data in a random-effects meta-

Figure 3. E-cigarette use and subsequent smoking cessation in cohort studies, adjusted analyses

Odds Ratio

Study or Subgroup log[Odds Ratio] SE Weight IV, Random, $95 \% \mathrm{Cl}$

$\begin{array}{lrrrr}\text { Exposure measured at baseline } & & & \\ \text { Al-Delaimy 2015 } & -0.8916 & 0.42 & 5.4 \% & 0.41[0.18,0.93] \\ \text { Brose 2015 } & -0.3147 & 0.2139 & 7.7 \% & 0.73[0.48,1.11] \\ \text { Gomajee 2019 } & 0.7395 & 0.0797 & 8.9 \% & 2.09[1.79,2.45] \\ \text { Grana 2014 } & -0.2744 & 0.3812 & 5.8 \% & 0.76[0.36,1.60] \\ \text { Hair 2018 } & -0.3978 & 0.0872 & 8.9 \% & 0.67[0.57,0.80] \\ \text { Mantey 2017 } & 0.131 & 0.1807 & 8.1 \% & 1.14[0.80,1.62] \\ \text { Manzoli 2017 } & 0.2231 & 0.1968 & 7.9 \% & 1.25[0.85,1.84] \\ \text { Pasquereau 2017 } & 0.1823 & 0.2069 & 7.8 \% & 1.20[0.80,1.80] \\ \text { Verplaetse 2019 } & -0.0202 & 0.0848 & 8.9 \% & 0.98[0.83,1.16] \\ \text { Weaver 2018 } & -1.204 & 0.4267 & 5.3 \% & 0.30[0.13,0.69] \\ \text { Subtotal (95\% Cl) } & & & 74.8 \% & 0.90[0.63,1.27]\end{array}$

Heterogeneity: $\mathrm{Tau}^{2}=0.26 ; \mathrm{Chi}^{2}=119.20, \mathrm{df}=9(\mathrm{P}<0.00001) ; \mathrm{I}^{2}=92 \%$

Test for overall effect: $Z=0.62(P=0.54)$

\section{Exposure measured at follow-up}

$\begin{array}{lrrrr}\text { Berry 2019b } & 0.7031 & 0.2056 & 7.8 \% & 2.02[1.35,3.02] \\ \text { Biener 2015 } & 0.5878 & 0.6639 & 3.3 \% & 1.80[0.49,6.61] \\ \text { Sutfin 2015 } & -0.9083 & 0.3218 & 6.5 \% & 0.40[0.21,0.76] \\ \text { Zhuang 2016 } & 0.157 & 0.2269 & 7.6 \% & 1.17[0.75,1.83] \\ \text { Subtotal (95\% Cl) } & & & 25.2 \% & 1.12[0.54,2.30]\end{array}$

Heterogeneity: $\mathrm{Tau}^{2}=0.42 ; \mathrm{Chi}^{2}=18.20, \mathrm{df}=3(\mathrm{P}=0.0004) ; \mathrm{I}^{2}=84 \%$

Test for overall effect: $Z=0.30(P=0.77)$

Total $(95 \% \mathrm{Cl})$ $100.0 \% \quad 0.95[0.70,1.28]$

Heterogeneity: $\mathrm{Tau}^{2}=0.25 ; \mathrm{Chi}^{2}=138.14, \mathrm{df}=13(\mathrm{P}<0.00001) ; \mathrm{I}^{2}=91 \%$

Test for overall effect: $Z=0.35(P=0.73)$

Test for subgroup differences: $\mathrm{Chi}^{2}=0.28, \mathrm{df}=1(\mathrm{P}=0.59), \mathrm{I}^{2}=0 \%$
Odds Ratio IV, Random, $95 \% \mathrm{Cl}$ 
analysis, the pooled odds ratio for smoking cessation was 1.78 (95\% CI: 1.41-2.25) for e-cigarette users compared with non-users (Figure 4).

Stratified analyses of studies with a short ( $\leq 6$ months) or a long ( $>6$ months) follow-up time (Supplementary file Figure S4), and studies among adults (aged $\geq 18$ years) (Supplementary file Figure S5) showed similar results. Studies stratified by sex or that included adolescents (aged $<18$ years) were not available.

\section{Certainty of evidence - RCTs}

The strength of the evidence concerning the association between e-cigarette use and smoking cessation was deemed to be low $(\oplus \oplus)$ due to risk of bias in any of the categories: randomization, deviation from planned intervention, attrition, measurement of outcome or reporting. Other reasons for downgrading were inconsistency between studies, mainly regarding differences in control alternatives, or that there was a lack of blinding, short follow-up time, limited number of studies, or small sample size (Table 4).

\section{Smoking cessation for at least 30 days among e-cigarette users and non-users}

For the more strict outcome of self-reported smoking cessation for at least 30 days, 15 cohort studies presented in 17 articles ${ }^{21,22,25,27,29-33,37-41,47,48}$, and four RCTs were included ${ }^{51,53,56,57}$, of which one is presented narratively ${ }^{57}$.

\section{Cohort studies}

Nine studies, with a total of 13588 participants, present

Table 3. E-cigarette use and subsequent smoking cessation in cohort studies

\begin{tabular}{|c|c|c|c|c|c|c|}
\hline $\begin{array}{l}\text { Association with } \\
\text { smoking cessation }\end{array}$ & Participants & $\begin{array}{l}\text { Number } \\
\text { of studies } \\
\text { (adjusted) }\end{array}$ & $\begin{array}{l}\text { Risk difference } \\
\text { RD }(95 \% \mathrm{CI}) *\end{array}$ & $\begin{array}{l}\text { Odds ratio } \\
\text { OR }(95 \% \text { CI })\end{array}$ & $\begin{array}{l}\text { Certainty of } \\
\text { evidence }\end{array}$ & Downgrading \\
\hline Full material & 39147 & $18(14)$ & $-0.01(-0.03-0.02)$ & $\begin{array}{c}\text { OR } \\
0.99(0.78-1.33) \\
\text { AOR } \\
0.95(0.70-1.28)\end{array}$ & $\begin{array}{l}\text { Very low } \\
\qquad(\oplus)\end{array}$ & $\begin{array}{l}-1 \text { risk of bias } \\
-1 \text { inconsistency } \\
-1 \text { imprecision }^{\mathrm{d}, \mathrm{e}}\end{array}$ \\
\hline $\begin{array}{l}\text { Short follow-up } \\
\text { ( } \leq 6 \text { months) }\end{array}$ & 3474 & $3(4)$ & $0.03(0.00-0.05)$ & $\begin{array}{c}\text { OR } \\
1.49(1.04-2.13) \\
\text { AOR } \\
0.99(0.69-1.44)\end{array}$ & $\begin{array}{l}\text { Very low } \\
\qquad(\oplus)\end{array}$ & $\begin{array}{l}-1 \text { risk of bias }^{\mathrm{a}} \\
-1 \text { inconsistency }^{\mathrm{b}} \\
-1 \text { imprecision }^{\mathrm{e}}\end{array}$ \\
\hline $\begin{array}{l}\text { Long follow-up } \\
\text { (>6 months) }\end{array}$ & 36029 & $16(12)$ & $-0.01(-0.03-0.02)$ & $\begin{array}{c}\text { OR } \\
0.96(0.74-1.24) \\
\text { AOR } \\
0.96(0.70-1.33)\end{array}$ & $\begin{array}{l}\text { Very low } \\
\qquad(\oplus)\end{array}$ & $\begin{array}{l}-1 \text { risk of bias } \\
-1 \text { inconsistency } \\
-1 \text { imprecision } \\
\text { d,e }\end{array}$ \\
\hline$<18$ years & 331 & $3(0)$ & $-0.12(-0.25-0.01)$ & $\begin{array}{c}\text { OR } \\
0.93(0.40-2.14) \\
\text { AOR } \\
\text { Not available }\end{array}$ & $\begin{array}{l}\text { Very low } \\
\qquad(\oplus)\end{array}$ & $\begin{array}{l}-2 \text { risk of bias }{ }^{\mathrm{a}, \mathrm{f}} \\
-1 \text { material } \\
\text { with several }^{\text {wimitations }} \mathrm{s}^{\mathrm{b}, \mathrm{d}, \mathrm{g}, \mathrm{h}}\end{array}$ \\
\hline$\geq 18$ years & 35275 & $14(12)$ & $-0.01(-0.03-0.01)$ & $\begin{array}{c}\text { OR } \\
0.96(0.73-1.25) \\
\text { AOR } \\
0.96(0.70-1.33)\end{array}$ & $\begin{array}{l}\text { Very low } \\
\qquad(\oplus)\end{array}$ & $\begin{array}{l}-1 \text { risk of bias } \\
-1 \text { inconsistency } \\
-1 \text { imprecision }^{\mathrm{d}, \mathrm{e}}\end{array}$ \\
\hline Women & 0 & $0(1)$ & Not available & $\begin{array}{c}\text { OR } \\
\text { Not available } \\
\text { AOR } \\
0.94(0.74-1.19)\end{array}$ & $\begin{array}{l}\text { Very low } \\
\qquad(\oplus)\end{array}$ & $\begin{array}{l}-1 \text { risk of bias }^{\mathrm{a}} \\
-1 \text { imprecision } \\
-1 \text { material with } \\
\text { several limitations }\end{array}$ \\
\hline Men & 0 & $0(1)$ & Not available & $\begin{array}{c}\text { OR } \\
\text { Not available } \\
\text { AOR } \\
1.00(0.79-1.27)\end{array}$ & $\begin{array}{l}\text { Very low } \\
\qquad(\oplus)\end{array}$ & $\begin{array}{l}-1 \text { risk of bias }^{\mathrm{a}} \\
-1 \text { imprecision } \\
-1 \text { material with } \\
\text { several limitations }\end{array}$ \\
\hline
\end{tabular}

${ }^{*}$ Calculated from unadjusted values. OR: unadjusted odds ratio. AOR: adjusted odds ratio. a Material with several deficits and limitations. $b$ The confidence intervals of individual studies include 1.0, indicating no statistically significant association. c The odds ratios of individual studies show associations in different directions. $d$ The pooled estimate for the unadjusted results includes 1.0, indicating no statistically significant association. e The pooled estimate for the adjusted results includes 1.0, indicating no statistically significant association. $\mathrm{f}$ Unadjusted analyses are not available. $\mathrm{g}$ The analysis is based on a limited number of studies. $\mathrm{h}$ The analysis is based on few participants. 
Figure 4. E-cigarette use and subsequent smoking cessation in randomized controlled trials

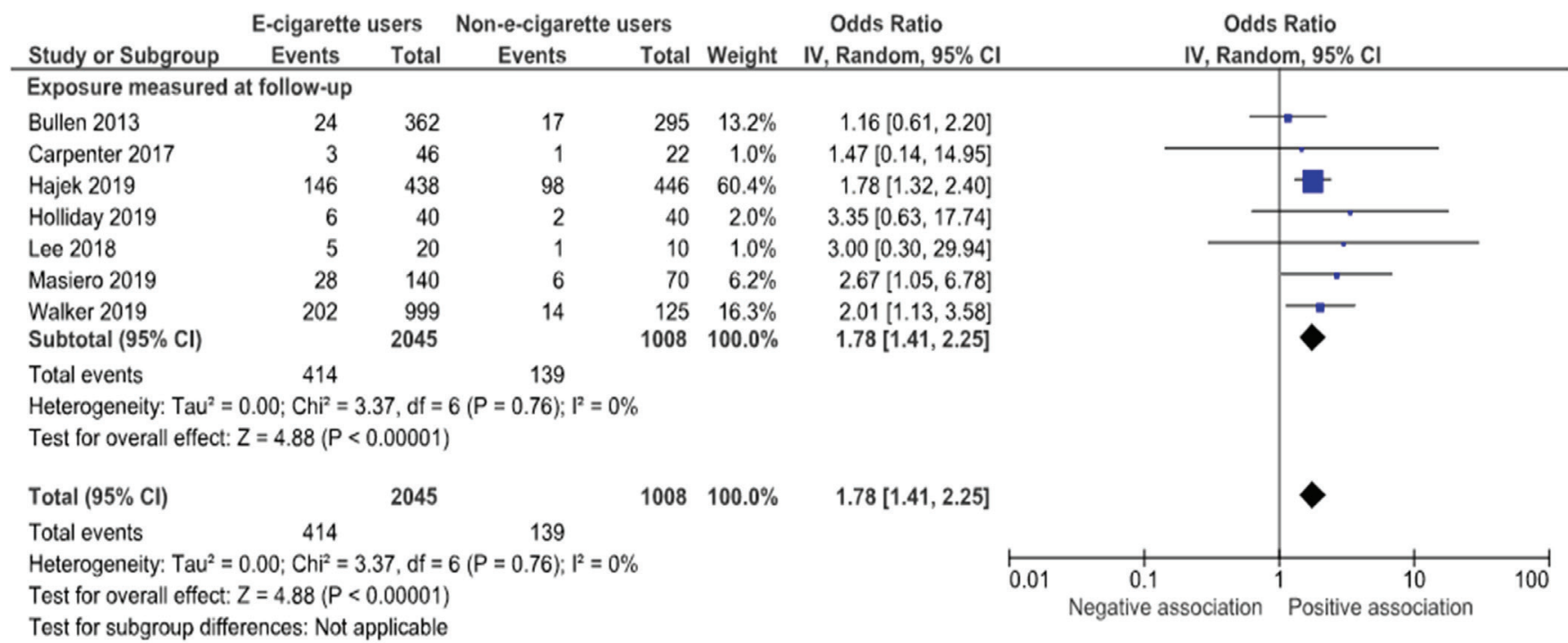

Meta-analysis of odds of smoking cessation among e-cigarette users compared with non-e-cigarette users.

Table 4. E-cigarette use and subsequent smoking cessation in randomized controlled trials

\begin{tabular}{|c|c|c|c|c|c|c|}
\hline $\begin{array}{l}\text { Association with } \\
\text { smoking cessation }\end{array}$ & Participants & $\begin{array}{l}\text { Number of } \\
\text { studies }\end{array}$ & $\begin{array}{l}\text { Risk difference } \\
\text { RD }(95 \% \text { CI })^{*}\end{array}$ & $\begin{array}{l}\text { Odds ratio } \\
\text { OR }(95 \% \mathrm{CI})\end{array}$ & $\begin{array}{l}\text { Certainty of } \\
\text { evidence }\end{array}$ & Down rating \\
\hline Full material & 3203 & $8^{* *}$ & $0.07(0.03-0.12)$ & $1.78(1.41-2.25)$ & $\begin{array}{l}\text { Low } \\
(\oplus \oplus)\end{array}$ & $\begin{array}{l}-1 \text { risk of bias }{ }^{\mathrm{a}} \\
-1 \text { inconsistency }\end{array}$ \\
\hline $\begin{array}{l}\text { Short follow-up } \\
\text { ( } \leq 6 \text { months) }\end{array}$ & 3203 & $8^{* *}$ & $0.06(0.02-0.10)$ & $1.67(1.32-2.11)$ & $\begin{array}{l}\text { Low } \\
(\oplus \oplus)\end{array}$ & $\begin{array}{l}-1 \text { risk of bias } \\
-1 \text { inconsistency }\end{array}$ \\
\hline $\begin{array}{l}\text { Long follow-up } \\
\text { (>6 months) }\end{array}$ & 884 & 1 & $0.09(0.04-0.14)$ & $2.00(1.38-2.89)$ & $\begin{array}{l}\text { Low } \\
(\oplus \oplus)\end{array}$ & $\begin{array}{l}-1 \text { risk of bias } \\
-1 \text { material with } \\
\text { several limitations }\end{array}$ \\
\hline$<18$ years & Not available & & & & & \\
\hline$\geq 18$ years & 3203 & $8^{* *}$ & $0.07(0.03-0.12)$ & $1.78(1.41-2.25)$ & $\begin{array}{l}\text { Low } \\
(\oplus \oplus)\end{array}$ & $\begin{array}{l}-1 \text { risk of bias }{ }^{a} \\
-1 \text { inconsistency }\end{array}$ \\
\hline Women & Not available & & & & & \\
\hline Men & 150 & $1^{* *}$ & Not available & Not available & $\begin{array}{l}\text { Low } \\
(\oplus \oplus)\end{array}$ & $\begin{array}{l}-1 \text { risk of bias }{ }^{\mathrm{a}} \\
-1 \text { material with } \\
\text { several limitations }{ }^{\mathrm{c}, \mathrm{d}}\end{array}$ \\
\hline
\end{tabular}

${ }^{*}$ Calculated from unadjusted values. ${ }^{* *}$ One study was included narratively. Outcome: 7-day point prevalence of abstinence after 24 weeks. Among participants allocated nicotine gum 29.3\% were abstinent, and 22.7\% among those allocated e-cigarettes. No statistically significant difference between groups. a Material with several deficits and limitations. $\mathrm{b}$ The confidence intervals of individual studies include 1.0, indicating no statistically significant association. $\mathrm{c}$ The analysis is based on a limited number of studies. $d$ The analysis is based on few participants.

unadjusted data (Figure 5). The odds ratios ranged between 0.30 and 1.51 for studies that measured e-cigarette use at baseline, and 1.02 to 1.20 for studies that measured e-cigarette use retrospectively at follow-up. The pooled unadjusted odds ratio for smoking cessation for at least 30 days was 0.76 (95\% CI: 0.45-1.26) and 1.05 (95\% CI: 0.93-1.19), respectively. Adjusted data were presented in 10 studies. The data were adjusted for sex $(9 / 10)$, age $(9 / 10)$ and socioeconomic factors $(8 / 10)$, but there was a large heterogeneity with respect to how these adjustments were made. The pooled adjusted odds ratio was 0.73 (95\% CI: 0.48-1.10) and 1.12 (95\% CI: 0.54-2.30), respectively (Figure 6). 
Figure 5. E-cigarette use and subsequent smoking cessation for at least 30 days in cohort studies, unadjusted analyses

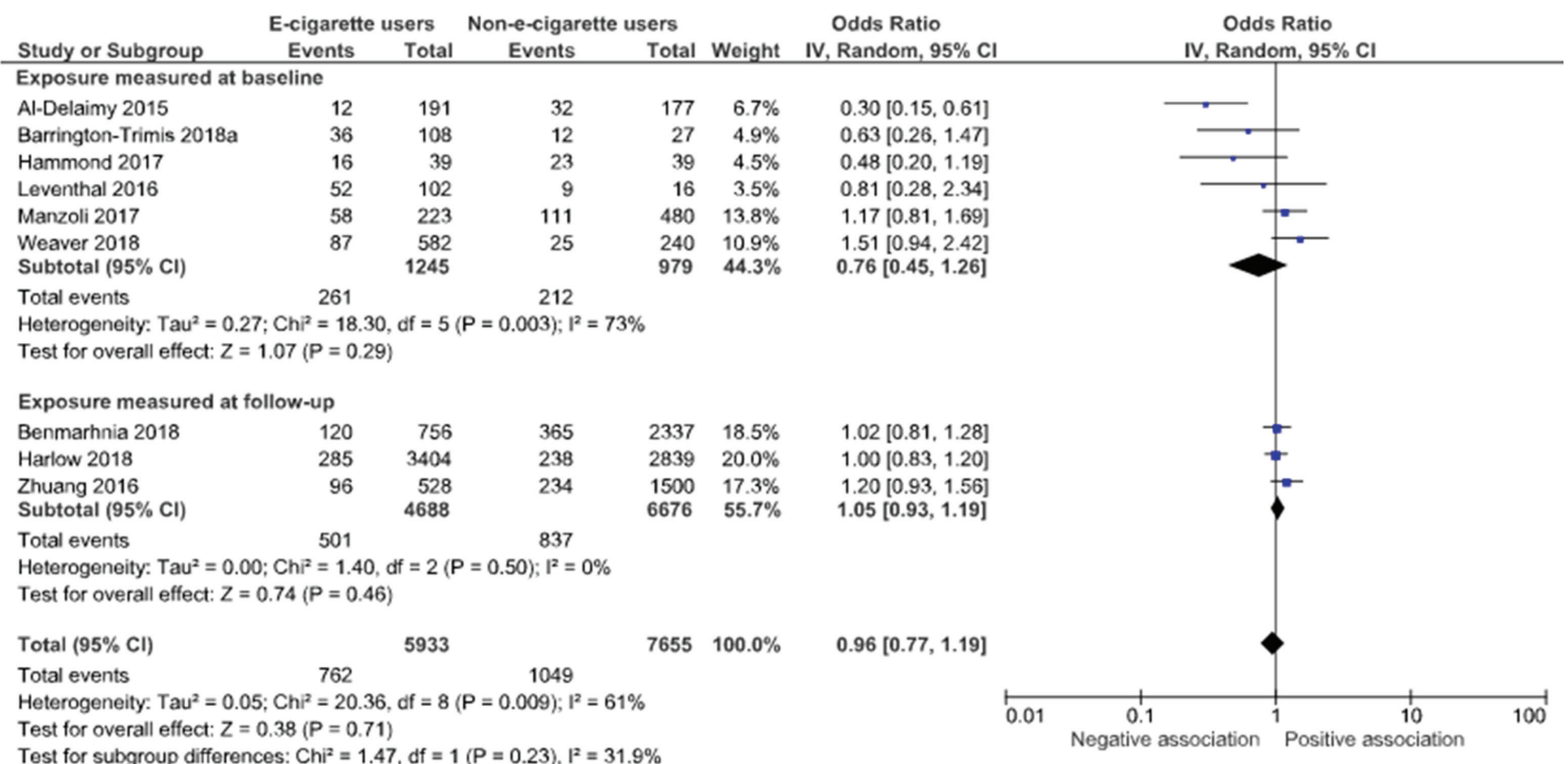

Meta-analysis of unadjusted odds of smoking cessation among e-cigarette users compared with non-e-cigarette users. Data are based on a total of 13588 participants, from 9 non-overlapping studies of low or medium risk of bias.

Figure 6. E-cigarette use and smoking cessation for at least 30 days in cohort studies, adjusted analyses

Odds Ratio

Odds Ratio

Study or Subgroup log[Odds Ratio] SE Weight IV, Random, $95 \% \mathrm{Cl}$ IV, Random, $95 \% \mathrm{Cl}$

$\begin{array}{lrrrr}\text { Exposure measured at baseline } & & & \\ \text { Al-Delaimy 2015 } & -0.8916 & 0.42 & 8.9 \% & 0.41[0.18,0.93] \\ \text { Hair 2018 } & -0.3978 & 0.0872 & 14.8 \% & 0.67[0.57,0.80] \\ \text { Manzoli 2017 } & 0.2231 & 0.1968 & 13.2 \% & 1.25[0.85,1.84] \\ \text { Pasquereau 2017 } & 0.0953 & 0.2306 & 12.5 \% & 1.10[0.70,1.73] \\ \text { Weaver 2018 } & -1.204 & 0.4267 & 8.8 \% & 0.30[0.13,0.69] \\ \text { Subtotal (95\% Cl) } & & & 58.2 \% & 0.73[0.48,1.10]\end{array}$

Heterogeneity: $\mathrm{Tau}^{2}=0.15 ; \mathrm{Chi}^{2}=17.73, \mathrm{df}=4(\mathrm{P}=0.001) ; \mathrm{I}^{2}=77 \%$

Test for overall effect: $Z=1.51(P=0.13)$

Exposure measured at follow-up

$\begin{array}{lrrrr}\text { Berry 2019b } & 0.7031 & 0.2056 & 13.0 \% & 2.02[1.35,3.02] \\ \text { Biener 2015 } & 0.5878 & 0.6639 & 5.4 \% & 1.80[0.49,6.61] \\ \text { Sutfin 2015 } & -0.9083 & 0.3218 & 10.7 \% & 0.40[0.21,0.76] \\ \text { Zhuang 2016 } & 0.157 & 0.2269 & 12.6 \% & 1.17[0.75,1.83] \\ \text { Subtotal }(95 \% \mathrm{Cl}) & & & 41.8 \% & 1.12[0.54,2.30]\end{array}$

Heterogeneity: $\mathrm{Tau}^{2}=0.42 ; \mathrm{Chi}^{2}=18.20, \mathrm{df}=3(\mathrm{P}=0.0004) ; \mathrm{I}^{2}=84 \%$

Test for overall effect: $Z=0.30(P=0.77)$

Total $(95 \% \mathrm{Cl})$ $100.0 \% \quad 0.86[0.59,1.25]$

Heterogeneity: $\mathrm{Tau}^{2}=0.24 ; \mathrm{Chi}^{2}=47.60, \mathrm{df}=8(\mathrm{P}<0.00001) ; \mathrm{I}^{2}=83 \%$

Test for overall effect: $Z=0.81(P=0.42)$

Test for subgroup differences: $\mathrm{Chi}^{2}=1.02, \mathrm{df}=1(\mathrm{P}=0.31), \mathrm{I}^{2}=2.1 \%$

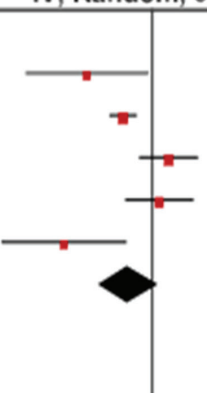

Meta-analysis of adjusted odds of smoking cessation among e-cigarette users compared with non-e-cigarette users. Studies were adjusted for sex (9/10), age (9/10), and socioeconomic factors (8/10). Data are from 9 non-overlapping studies of low or medium risk of bias. 
Figure 7. E-cigarette use and subsequent smoking cessation for at least 30 days in randomized controlled trials

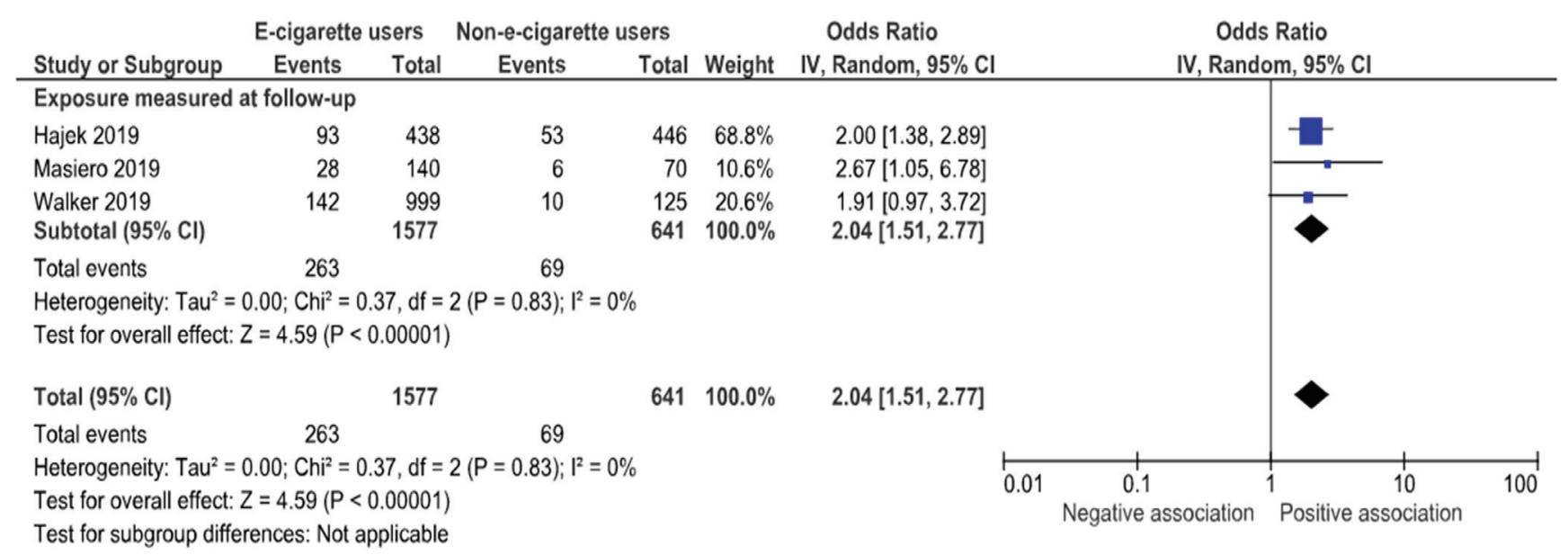

Meta-analysis of odds of smoking cessation among e-cigarette users compared with non-e-cigarette users.

Stratified analysis of studies with a short $(\leq 6$ months) or a long (>6 months) follow-up time (Supplementary file Figure S6), and studies among adolescents (aged $<18$ years) or adults (aged $\geq 18$ years) showed a similar result (Supplementary file Figure S7). Stratified data by sex or adjusted analyses among adolescents were not available.

\section{Certainty of evidence - cohort studies}

The strength of the evidence concerning the association between e-cigarette use and smoking cessation for at least 30 days in cohort studies was deemed to be very low $(\oplus)$ due to risk of bias in any of the categories: selection, exposure, judgement or reporting, heterogeneity or imprecision between studies, or that the material was based on few studies or few participants (Supplementary file Table S7).

\section{Randomized controlled trials}

Three studies, with a total of 2218 participants were included in the meta-analysis. One study was included narratively, as it presents the proportion of participants that achieved smoking cessation lasting from the 9 th until the 24th week of the study ${ }^{57}$. All included RCTs showed a positive association between e-cigarette use and smoking cessation for at least 30 days and the odds ratios ranged between 1.91 to 2.67 for e-cigarette users compared with non-users. The pooled odds ratio was 2.04 (95\% CI: 1.51-2.77)
(Figure 7).

Stratified analysis of studies with a short $(\leq 6$ months) or a long ( $>6$ months) follow-up time (Supplementary file Figure S8), and studies among adults (aged $\geq 18$ years) showed a similar result (Supplementary file Figure S9). Studies presenting data of adolescents (aged $<18$ years) or women, were not available. One study was performed only among men, and was included narratively (Supplementary file Table S7).

\section{Certainty of evidence - RCTs}

The strength of the evidence concerning the association between e-cigarette use and smoking cessation for at least 30 days in RCTs was deemed to be low $(\oplus \oplus)$ due to risk of bias in any of the categories: randomization, deviation from planned intervention, attrition, measurement of outcome, or reporting. Other reasons for downgrading were a limited number of studies and small sample size (Supplementary file Table S7).

\section{Assessment of publication bias}

Funnel plots did not indicate any publication bias in the material (Supplemental file Figure S10 A-C).

We did not find quality evidence for an association between e-cigarette use and smoking cessation. Although RCTs tended to support a more positive association between e-cigarette use and smoking 
cessation than the cohort studies, the grading of evidence was consistently low.

\section{DISCUSSION}

In this systematic review and meta-analysis, including both observational studies with a longitudinal design and experimental studies, we did not find quality evidence for an association between e-cigarette use and smoking cessation. However, the direction of the association differed between observational and experimental studies. In fact, while the pooled estimates from observational studies were close to the null, those from RCTs indicated in all cases that the use of e-cigarettes compared to control situations was associated with higher likelihood of smoking cessation $(70-80 \%$ in the pooled results). In one of the few available systematic reviews that distinguished between observational studies and RCTs, the authors concluded that e-cigarette use was associated with smoking cessation in RCTs but was not associated with smoking cessation in the general adult population $^{58}$. Based on these findings, the authors suggest that e-cigarettes should not be approved as a consumer product, but they could be considered as a prescription therapy for smoking cessation.

The different results between observational studies and RCTs can be explained by several features. First, controlling for confounders is stricter in RCTs than in observational studies. Other than sex, age and socioeconomic factors, there are many other confounders that may affect the association between e-cigarette use and smoking cessation in observational studies. There are a wide range of reasons for initiating e-cigarette use, for instance to quit smoking, curiosity, the different flavors, lower price than tobacco cigarettes, or due to smoking bans $^{59}$. Moreover, smoking cessation is associated with several physiological, psychological and social factors such as strength of nicotine dependence, withdrawal symptoms, fear of failure, readiness to make a quit attempt, lack of support, and being around other smokers ${ }^{60}$. These factors are rarely taken into account in observational studies and may contribute to the different associations with the outcome. For RCTs on the other hand, the vast majority of the studies only included smokers that were motivated to quit. Thus, if the smokers were equally randomized into intervention and control group, the motivation to quit would be the same between the groups and any difference in smoking cessation would be more strongly related to e-cigarette use. However, being motivated to quit could increase the likelihood of a successful outcome enhanced by the use of e-cigarettes and may not be generalizable to smokers in the general population. Nevertheless, in line with a systematic literature review by the Cochrane Library ${ }^{7}$, we rated the evidence of an association between e-cigarette use and smoking cessation in the RCTs as low, mainly due to limitations in the study design, sample size or choice of control conditions.

Another explanation may be that misclassification of exposure, in this case e-cigarette use, is more common in observational studies. Among those defined as e-cigarette users in the observational studies, we can assume a large variation for instance in the type of e-cigarette used, composition of the e-liquid, level of nicotine, e-cigarette use behavior, and how long they have been an e-cigarette user. On the other hand, the assessment of these features of e-cigarette use and nicotine exposure is complicated by the fact that many e-cigarette users are unaware of the content in nicotine in the e-cigarettes they are using, and it has been shown that the level of nicotine is sometimes incorrectly labelled on the product ${ }^{61}$. Moreover, there is no standardized method of reporting the level of e-cigarette exposure, at odds with number of cigarettes or packyears for tobacco cigarette smoking. Therefore, the distinction between different e-cigarette uses or nicotine exposure in relation to smoking cessation is seldom possible.

Although most cohort studies measured e-cigarette use at baseline, some obtained that information retrospectively at the follow-up survey. In the latter studies, the sequential order of e-cigarette use and smoking cessation cannot be fully assessed. It is possible that the participants quit smoking first and then initiated e-cigarette use. Therefore, we chose to present the analyses separately for these two groups of studies. However, regardless of when the exposure was measured, the analyses showed similar results, not supporting the presence of an association between e-cigarette use and smoking cessation in population studies.

While RCTs provide higher level of evidence 
and are more reliable in the exploration of a causal link, observational studies are more informative on the potential impact of e-cigarettes at a population level. Taken together, we conclude that although e-cigarettes may favor smoking cessation over and above a number of conditions among motivated smokers, their impact at the population level, if any, would likely be small.

\section{Strengths and limitations}

The major strength of this review is that it distinguishes between cohort studies and RCTs and bases the conclusion on studies using a longitudinal prospective design. Furthermore, to avoid inclusion of articles that were subject to major limitations our review only included prospective studies with a low or moderate risk of bias and duration of follow-up of at least three months. Limitations were the considerable heterogeneity between studies and measures of e-cigarette use. Most studies did not assess the type of device used, how often the e-cigarette was used, the composition of e-liquid, or whether e-cigarettes contained nicotine or not. The adjustment for potential confounding factors was very different across studies. It was thus not possible to grade the effect size of the association. Grading of evidence thereby relates to the certainty of evidence for the association between e-cigarette use and smoking cessation. The drop-out rate was relatively high in most studies, therefore limiting the statistical power and increasing the potential for selection bias. In this review, we did not exclude articles due to their attrition, but this aspect was considered in the judgment of the risk of bias and in the grading of evidence.

\section{CONCLUSIONS}

This review and meta-analysis could not find quality evidence for an association between e-cigarette use and smoking cessation. Although RCTs tended to support a more positive association between e-cigarette use and smoking cessation than the cohort studies, the grading of evidence was consistently low.

\section{REFERENCES}

1. Lundbäck B, Katsaounou P, Lötvall J. The up-rise in e-cigarette use - friend or foe? Respir Res. 2016;17(1):52. doi:10.1186/s12931-016-0371-2

2. Farsalinos K. E-cigarettes: An aid in smoking cessation, or a new health hazard? Ther Adv Respir Dis. 2018;12:1-20. doi:10.1177/1753465817744960

3. Bhatt JM, Ramphul M, Bush A. An update on controversies in e-cigarettes. Paediatr Respir Rev. 2020;36:75-86 doi:10.1016/j.prrv.2020.09.003

4. Nutt DJ, Phillips LD, Balfour D, et al. Estimating the harms of nicotine-containing products using the MCDA approach. Eur Addict Res. 2014;20(5):218-225. doi:10.1159/000360220

5. Notley C, Ward E, Dawkins L, Holland R. The unique contribution of e-cigarettes for tobacco harm reduction in supporting smoking relapse prevention. Harm Reduct J. 2018;15(1):31. doi:10.1186/s12954-018-0237-7

6. Soneji S, Barrington-Trimis JL, Wills TA, et al. Association between initial use of e-cigarettes and subsequent cigarette smoking among adolescents and young adults a systematic review and meta-analysis. JAMA Pediatrics. 2017;171(8):788797. doi:10.1001/jamapediatrics.2017.1488

7. Hartmann-Boyce J, McRobbie H, Lindson N, et al. Electronic cigarettes for smoking cessation. Cochrane Database Syst Rev. 2020;10(10):CD010216. doi:10.1002/14651858.CD010216.pub5

8. Pisinger C, Dagli E, Filippidis FT, et al. ERS and tobacco harm reduction. Eur Respir J. 2019;54(6):1902009. doi:10.1183/13993003.02009-2019

9. Mcrobbie H, Bullen C, Hartmann-Boyce J, Hajek P. Electronic cigarettes for smoking cessation and reduction. Cochrane Database Syst Rev. 2014;(12):CD010216. doi:10.1002/14651858.CD010216.pub2

10. Rahman MA, Hann N, Wilson A, Mnatzaganian G, Worrall-Carter L. E-Cigarettes and smoking cessation: Evidence from a systematic review and meta-analysis. PLoS One. 2015;10(3):e0122544. doi:10.1371/journal.pone.0122544

11. Kalkhoran S, Glantz SA. E-cigarettes and smoking cessation in real-world and clinical settings: A systematic review and meta-analysis. Lancet Respir Med. 2016;4(2):116-128. doi:10.1016/S2213-2600(15)00521-4

12. El Dib R, Suzumura EA, Akl EA, et al. Electronic nicotine delivery systems and/or electronic non-nicotine delivery systems for tobacco smoking cessation or reduction: A systematic review and meta-analysis. BMJ Open. 2017;7(2):e012680. doi:10.1136/bmjopen-2016-012680

13. Malas M, van der Tempel J, Schwartz R, et al. Electronic cigarettes for smoking cessation: A systematic review. Nicotine Tob Res. 2016;18(10):1926-1936. doi:10.1093/ntr/ntw119

14. Hartmann-Boyce J, Mcrobbie H, Bullen C, Begh R, Stead LF, Hajek P. Electronic cigarettes for smoking cessation. Cochrane Database Syst Rev. 2020;10(10):CD010216. doi:10.1002/14651858.CD010216.pub3

15. Swedish Agency for Health Technology Assessment and Assessment of Social Services. Associations between the use of snus (moist tobacco) or electronic cigarettes and tobacco smoking. SBU; 2020. SBU 2020/312E. Accessed May 1, 2021. https://www.sbu.se/en/publications/sbu- 
bereder/associations-between-the-use-of-snus-moisttobacco-or-electronic-cigarettes-and-tobacco-smoking/

16. Cochrane. Cochrane Handbook for Systematic Reviews of Interventions. Accessed August 26, 2021. https:// training.cochrane.org/handbook

17. Atkins D, Best D, Briss P, et al. Grading quality of evidence and strength of recommendations. Br Med J. 2004;328:1490-1494. doi:10.1136/bmj.328.7454.1490

18. Guyatt GH, Oxman AD, Vist GE, et al. GRADE: An emerging consensus on rating quality of evidence and strength of recommendations. BMJ. 2008;336(7650):924926. doi:10.1136/bmj.39489.470347.ad

19. Morgan RL, Thayer KA, Santesso N, et al. A risk of bias instrument for non-randomized studies of exposures: A users' guide to its application in the context of GRADE. Environ Int. 2019;122:168-184. doi:10.1016/j.envint.2018.11.004

20. Schünemann HJ, Cuello C, Akl EA, et al. GRADE guidelines: 18. How ROBINS-I and other tools to assess risk of bias in nonrandomized studies should be used to rate the certainty of a body of evidence. J Clin Epidemiol. 2019;111:105-114. doi:10.1016/j.jclinepi.2018.01.012

21. Barrington-Trimis JL, Bello MS, Liu F, et al. Ethnic Differences in Patterns of Cigarette and E-Cigarette Use Over Time Among Adolescents. J Adolesc Heal. 2019;65(3):359365. doi:10.1016/j.jadohealth.2019.04.002

22. Barrington-Trimis JL, Kong G, Leventhal AM, et al. E-cigarette use and subsequent smoking frequency among adolescents. Pediatrics. 2018;142(6):e20180486. doi:10.1542/peds.2018-0486

23. Kurti AN, Bunn JY, Villanti AC, et al. Patterns of single and multiple tobacco product use among us women of reproductive age. Nicotine Tob Res. 2018;20(Suppl 1):S71-S80. doi:10.1093/ntr/nty024

24. Mantey DS, Cooper MR, Loukas A, Perry CL. E-cigarette use and cigarette smoking cessation among Texas college students. Am J Health Behav. 2017;41(6):750-759. doi:10.5993/AJHB.41.6.9

25. Sutfin EL, Reboussin BA, Debinski B, Wagoner KG, Spangler J, Wolfson M. The impact of trying electronic cigarettes on cigarette smoking by college students: A prospective analysis. Am J Public Health. 2015;105(8):e83-e89. doi:10.2105/AJPH.2015.302707

26. Verplaetse TL, Moore KE, Pittman BP, et al. Intersection of E-Cigarette Use and Gender on Transitions in Cigarette Smoking Status: Findings Across Waves 1 and 2 of the Population Assessment of Tobacco and Health Study. Nicotine Tob Res. 2019;21(10):1423-1428. doi:10.1093/ntr/nty187

27. Weaver SR, Huang J, Pechacek TF, Heath JW, Ashley DL, Eriksen MP. Are electronic nicotine delivery systems helping cigarette smokers quit? Evidence from a prospective cohort study of U.S. adult smokers, 2015-2016. PLoS One. 2018;13(7):2015-2016. doi:10.1371/journal.pone.0198047
28. Piper ME, Baker TB, Benowitz NL, Jorenby DE. Changes in Use Patterns over 1 Year among Smokers and Dual Users of Combustible and Electronic Cigarettes. Nicotine Tob Res. 2020;22(5):672-680. doi:10.1093/ntr/ntz065

29. Zhuang YL, Cummins SE, Sun JY, Zhu SH. Long-term E-cigarette use and smoking cessation: A longitudinal study with US population. Tob Control. 2016;25(Suppl 1):i90-i95. doi:10.1136/tobaccocontrol-2016-053096

30. Leventhal AM, Angeles L, Stone MD, et al. Association of e-cigarette vaping and progression to heavier patterns of cigarette smoking. JAMA. 2017;316(18):1918-1920. doi:10.1001/jama.2016.14649

31. Biener L, Lee Hargraves J. A longitudinal study of electronic cigarette use among a population-based sample of adult smokers: Association with smoking cessation and motivation to quit. Nicotine Tob Res. 2015;17(2):127133. doi:10.1093/ntr/ntu200

32. Berry KM, Reynolds LM, Collins JM, et al. E-cigarette initiation and associated changes in smoking cessation and reduction: The Population Assessment of Tobacco and Health Study, 2013-2015. Tob Control. 2019;28:42-49. doi:10.1136/tobaccocontrol-2017-054108

33. Hair EC, Romberg AR, Niaura R, et al. Longitudinal tobacco use transitions among adolescents and young adults: 2014-2016. Nicotine Tob Res. 2019;21(4):458468. doi:10.1093/ntr/ntx285

34. Chen JC. Flavored E-cigarette Use and Cigarette Smoking Reduction and Cessation-A Large National Study among Young Adult Smokers. Subst Use Misuse. 2018;53(12):20172031. doi:10.1080/10826084.2018.1455704

35. Curry E, Nemeth JM, Wermert A, et al. A Descriptive Report of Electronic Cigarette Use After Participation in a Community-Based Tobacco Cessation Trial. Nicotine Tob Res. 2017;20(1):135-139. doi:10.1093/ntr/ntx013

36. Kasza KA, Borek N, Conway KP, et al. Transitions in tobacco product use by u.S. adults between 20132014 and 2014-2015: Findings from the path study wave 1 and wave 2 . Int J Environ Res Public Health. 2018;15(11):2013-2014. doi:10.3390/ijerph15112515

37. Leventhal AM, Strong DR, Kirkpatrick MG, et al. Association of electronic cigarette use with initiation of combustible tobacco product smoking in early adolescence. JAMA - J Am Med Assoc. 2015;314(7):700707. doi:10.1001/jama.2015.8950

38. Niaura R, Rich I, Johnson AL, et al. Young Adult Tobacco and E-cigarette Use Transitions: Examining Stability Using Multistate Modeling. Nicotine Tob Res. 2020;22(5):647-654. doi:10.1093/ntr/ntz030

39. Al-Delaimy WK, Myers MG, Leas EC, Strong DR, Hofstetter CR. E-cigarette use in the past and quitting behavior in the future: A population-based study. Am J Public Health. 2015;105(6):1213-1219. doi:10.2105/AJPH.2014.302482

40. Benmarhnia T, Pierce JP, Leas E, et al. Can E-Cigarettes and Pharmaceutical aids Increase Smoking Cessation 
and Reduce Cigarette Consumption? Findings from a Nationally Representative Cohort of American Smokers. Am J Epidemiol. 2018;187(11):2397-2404. doi:10.1093/aje/kwy129

41. Harlow AF, Stokes A, Brooks DR. Socioeconomic and Racial/Ethnic Differences in E-Cigarette Uptake among Cigarette Smokers: Longitudinal Analysis of the Population Assessment of Tobacco and Health (PATH) Study. Nicotine Tob Res. 2019;21(10):1385-1393. doi:10.1093/ntr/nty141

42. Grana RA, Popova L, Ling PM. A longitudinal analysis of electronic cigarette use and smoking cessation. JAMA Intern Med. 2014;174(5):812-813. doi:10.1001/jamainternmed.2014.187

43. Hammond D, Reid JL, Cole AG, Leatherdale ST. Electronic cigarette use and smoking initiation among youth: A longitudinal cohort study. CMAJ. 2017;189(43):E1328-E1336. doi:10.1503/cmaj.161002

44. Brose LS, Hitchman SC, Brown J, West R, McNeill A. Is the use of electronic cigarettes while smoking associated with smoking cessation attempts, cessation and reduced cigarette consumption? A survey with a 1-year follow-up. Addiction. 2015;110(7):1160-1168. doi:10.1111/add.12917

45. Flacco ME, Ferrante M, Fiore M, et al. Cohort study of electronic cigarette use: Safety and effectiveness after 4 years of follow-up. Eur Rev Med Pharmacol Sci. 2019;23(1):402-412. doi:10.26355/eurrev_201901_16789

46. Gomajee R, El-Khoury F, Goldberg M, et al. Association between Electronic Cigarette Use and Smoking Reduction in France. JAMA Intern Med. 2019;179(9):1193-1200. doi:10.1001/jamainternmed.2019.1483

47. Manzoli L, Flacco ME, Ferrante M, et al. Cohort study of electronic cigarette use: Effectiveness and safety at 24 months. Tob Control. 2017;26(3):284-292. doi:10.1136/tobaccocontrol-2015-052822

48. Pasquereau A, Guignard R, Andler R, Nguyen-Thanh V. Electronic cigarettes, quit attempts and smoking cessation: a 6-month follow-up. Addiction. 2017;112(9):1620-1628. doi:10.1111/add.13869

49. Carpenter MJ, Heckman BW, Wahlquist AE, et al. A naturalistic, randomized pilot trial of e-cigarettes: Uptake, exposure, and behavioral effects. Cancer Epidemiol Biomarkers Prev. 2017;26(12):1795-1803. doi:10.1158/1055-9965.EPI-17-0460

50. Lee SM, Tenney R, Wallace AW, Arjomandi M. E-cigarettes versus nicotine patches for perioperative smoking cessation: A pilot randomized trial. PeerJ. 2018;6:e5609. doi:10.7717/peerj.5609

51. Hajek P, Phillips-Waller A, Przulj D, et al. A Randomized Trial of E-Cigarettes versus Nicotine-Replacement Therapy. N Engl J Med. 2019;380(7):629-637. doi:10.1056/nejmoa1808779

52. Holliday R, Preshaw PM, Ryan V, et al. A feasibility study with embedded pilot randomised controlled trial and process evaluation of electronic cigarettes for smoking cessation in patients with periodontitis. Pilot Feasibility Stud. 2019;5(1):74. doi:10.1186/s40814-019-0451-4

53. Masiero M, Lucchiari C, Mazzocco K, et al. E-cigarettes may support smokers with high smoking-related risk awareness to stop smoking in the short run: Preliminary results by randomized controlled trial. Nicotine Tob Res. 2019;21(1):119-126. doi:10.1093/ntr/nty047

54. Bullen C, Howe C, Laugesen M, et al. Electronic cigarettes for smoking cessation: A randomised controlled trial. Lancet. 2013;382(9905):1629-1637. doi:10.1016/S0140-6736(13)61842-5

55. O'Brien B, Knight-West O, Walker N, Parag V, Bullen C. E-cigarettes versus NRT for smoking reduction or cessation in people with mental illness: Secondary analysis of data from the ASCEND trial. Tob Induc Dis. 2015;13(March):5. doi:10.1186/s12971-015-0030-2

56. Walker N, Mbchb B, Verbiest M, et al. Nicotine patches used in combination with e-cigarettes (with and without nicotine) for smoking cessation: a pragmatic, randomised trial. Lancet Respir Med. 2019;8(1):54-64. doi:10.1016/S2213-2600(19)30269-3

57. Lee SH, Ahn SH, Cheong YS. Effect of electronic cigarettes on smoking reduction and cessation in Korean Male smokers: A randomized controlled study. J Am Board Fam Med. 2019;32(4):567-574. doi:10.3122/jabfm.2019.04.180384

58. Wang RJ, Bhadriraju S, Glantz SA. E-cigarette use and adult cigarette smoking cessation: A metaanalysis. Am J Public Health. 2021;111(2):230-246. doi:10.2105/AJPH.2020.305999

59. Romijnders KAGJ, Osch L van, Vries H de, Talhout R. Perceptions and reasons regarding e-cigarette use among users and non-users: A narrative literature review. Int J Environ Res Public Health. 2018;15(6):1190. doi:10.3390/ijerph15061190

60. Martínez C, Schoretsaniti S, Bobak A, Fletcher M. Behaviour counselling and psychosocial interventions. The role of patient involvement. In: Supporting Tobacco Cessation. 2021;76-96. doi:10.1183/2312508x.10013220

61. DeVito EE, Krishnan-Sarin S. E-cigarettes: Impact of E-Liquid Components and Device Characteristics on Nicotine Exposure. Curr Neuropharmacol. 2018;16(4):438. doi:10.2174/1570159x15666171016164430

\section{ACKNOWLEDGEMENTS}

We acknowledge the assistance provided by Elisabeth Gustafsson, Martin Norman, Hanna Olofsson and Karin Stenström at SBU, Stockholm, Sweden.

\section{CONFLICTS OF INTEREST}

The authors have each completed and submitted an ICMJE Form for Disclosure of Potential Conflicts of Interest. The authors declare that they have no competing interests, financial or otherwise, related to the current work. M. R. Galanti reports that she is is employed by the Stockholm County Health Care Service. 


\section{FUNDING}

This project was initiated in collaboration between The Public Health Agency of Sweden and the Swedish Agency for Health Technology Assessment and Assessment of Social Services (SBU). LH was funded by The Swedish Heart-Lung Foundation (Proposal numbers: 20200765, 20200750, 20170696, 20170438), ALF by a regional agreement between Umeå University and Västerbotten County Council (Proposal number: RV-738451), and Norrbotten County Council (Proposal number: NLL-933163). LA was funded by the Swedish research council (Proposal number: 2018-0258). The funders had no role in study design, data collection and analysis, decision to publish or preparation of the manuscript.

ETHICAL APPROVAL AND INFORMED CONSENT

Ethical approval and informed consent were not required for this study.

DATA AVAILABILITY

The data supporting this research are available from the authors on reasonable request.

\section{AUTHORS' CONTRIBUTIONS}

All authors participated in the conceptualization of the work and interpretation of data. LH wrote the original draft and revised it after critical review. MRG revised and edited the manuscript. LR contributed to project administration, formal analysis, and visualization. $\mathrm{HG}$ and LA revised and edited the manuscript. All authors approved the final version to be published.

PROVENANCE AND PEER REVIEW

Not commissioned; externally peer reviewed. 